\title{
Physiological reactions to fear provocation in dogs
}

\author{
E Hydbring-Sandberg, L W von Walter, K Höglund, \\ K Svartberg ${ }^{1}$, L Swenson ${ }^{2}$ and B Forkman ${ }^{3}$
}

\author{
Department of Anatomy and Physiology, Swedish University of Agricultural Sciences, Box 7045, S-750 07 Uppsala, Sweden \\ ${ }^{1}$ Department of Zoology, Stockholm University, Stockholm, Sweden \\ ${ }^{2}$ Department of Animal Breeding and Genetics, Swedish University of Agricultural Sciences, Uppsala, Sweden \\ ${ }^{3}$ Department of Animal Science and Animal Health, Royal Veterinary and Agricultural University, DK-1870 Frederiksberg, Denmark \\ (Requests for offprints should be addressed to E Hydbring-Sandberg; Email: Eva.sandberg@afys.slu.se)
}

\begin{abstract}
Fear is a common behavioral problem in dogs. In this paper, we studied the association between behavioral and physiological responses in two potentially fear-eliciting situations. The aim was to establish whether it is possible to separate dogs of the collie breed that are fearful of floors and gunshots from those that are not by studying changes in heart rate and hematocrit, plasma cortisol, progesterone, testosterone, vasopressin, and $\beta$-endorphin concentrations. Thirteen privately owned male dogs of the collie breed were studied during a floor test, using different types of floors, and a subsequent gunshot test. Seven of the dogs were identified as being fearful of floors and six were declared as fearless. Out of the 13 dogs, seven were fearful of gunshots and six were fearless of gunshots. Since fear of floors did not always occur concomitantly with fear of gunshots, there were consequently four different groups of dogs. The heart rate increased during the floor test in all
\end{abstract}

groups, but dogs that were fearful of floors had higher heart rates than dogs that were fearless of floors. Dogs that were fearful of gunshots had higher heart rates, higher hematocrit levels and higher plasma concentrations of cortisol, progesterone, vasopressin, and $\beta$-endorphins during the gunshot test than did dogs that were found to be fearless of gunshots. Plasma cortisol and progesterone increased drastically during the gunshot test in dogs identified as being fearful of gunshots. In fearful dogs, the testosterone concentration increased after completion of the floor test and before the gunshot test started, but there were no significant differences in testosterone between the groups. Since dogs fearful of gunshots had increased levels of several physiological parameters, the results demonstrated that this fear is a serious stress for the individual, a fear which it is possible to register with physiological variables. Journal of Endocrinology (2004) 180, 439-448

\section{Introduction}

Behavioral problems in dogs have, during the past few decades, attracted increased attention. Besides aggressive behavior towards humans and other dogs, fear-related behavior is reported to be the most common problem (Blackshaw 1988, Beaver 1999, Lindsay 2001). Fear has been defined as an emotional state which is induced by the perception of danger that threatens the wellbeing of the individual (Gray 1987). Consequently, fearfulness can be said to be a normal self-protective response that helps the individual cope with a challenging environment. However, individual dogs differ in fearfulness, which implies that some dogs are more exposed than others (for example see Plutchik 1971). Furthermore, in modern society, there are a number of potential fear-eliciting stimuli and situations for which the dog may not be adapted (e.g. crowded areas, traffic, and fireworks). These factors enhance the risk of a maladaptive level of fear in certain dogs, which threatens the welfare of these animals.
Fear can be expressed in different ways in the dog. Behaviorally, the most commonly observed signs of fear in dogs are avoidance, flight, immobility, and aggressive behavior (Scott \& Fuller 1965, Fox 1978, King et al. 2003). These fear-related behaviors seem to be common to a range of species (Boissy 1995). It is well known that stress and fear activate several physiological systems in the organism. The sympathetic nervous system becomes activated, which stimulates the secretion of adrenaline and noradrenaline from the adrenal medulla. In addition, the sympathetic nervous system stimulates the spleen to release stored red blood cells, which increases the hematocrit level. In dogs, this hematocrit increase is pronounced (Reeve et al. 1953). In addition, activation of the sympathetic nervous system usually increases blood pressure and heart rate. Stress and fear also stimulate the hypothalamicpituitary-adrenal-cortical system, which may lead to elevations in the well-established stress hormone cortisol, a hormone associated with a passive strategy, loss of control, and depression (Henry 1993). Glucocorticoids usually 
facilitate the extinction of learned fear responses since their mode of action is to reduce fear, possibly by inhibiting adrenocorticotropic hormone (ACTH) (Giusto et al. 1971). In dogs, cortisol has been found to increase during transportation (Kuhn et al. 1991, Bergeron et al. 2002) and shock (Dess et al. 1983), when exposed to sound blasts (Beerda et al. 1998), and during social and spatial restriction (Beerda et al. 1999). In one study, it was reported that when ACTH was administered to dogs not only cortisol but also progesterone increased markedly in both males and females (Frank et al. 2003). This suggests that progesterone may be a further indicator of activation of the hypothalamic-pituitary axis.

The steroid testosterone has been measured in several stress studies. In human beings, testosterone has been reported to increase during aggression and anger (Christiansen \& Knussmann 1987, Dabbs 1993, Scerbo \& Kolko 1994, Van Honk et al. 1999), dominance (Christiansen \& Knussmann 1987), experiences of winning, for instance in sporting events (McCaul et al. 1992, Bernhardt et al. 1998), psychic stress (Christiansen et al. 1985), and violent and antisocial behavior (Dabbs 1993) as well as with sexual activity (Dabbs 1993). It has been reported to decrease during experiences of losing (Dabbs 1993, Bernhardt et al. 1998), anxiety (Davies et al. 1992), passive avoidant coping behavior (Müller 1994), and depression (Dabbs 1993).

One common, but not scientifically reported, explanation for the fear of gunshots in dogs is that dogs may feel pain in their ears during the gunshots. Therefore, we also measured the hormones vasopressin and $\beta$-endorphin, hormones associated with stress and pain relief (Fredrickson \& Geary 1982, Cameron et al. 1985). Opiates act synergistically as regulatory molecules and integrate most of the autonomic and metabolic responses to stress (Molina \& Abumrad 1994).

There are therefore a number of behavioral and physiological variables that are regarded to be indicators of stress in dogs in fear-related situations. For the valid interpretation of stress, it is of importance to use both behavioral and physiological measures (Beerda et al. 2000). In dogs, such methodology has been used in studies investigating fear-related stress in individuals exposed to stimuli such as sound blasts, electric shocks, and physical restraint (Beerda et al. 1998, King et al. 2003). However, to our knowledge, no studies on the fear of gunshots, or the fear of walking on different surfaces, such as different types of floors, have been reported in which both physiological and behavioral variables were measured. Fear of a loud noise, such as gunshot, is considered to be a common problem in dogs (Blackshaw 1988). Fear of walking on certain floors has been indicated in results from questionnaires answered by dog owners (L Swenson, unpublished data, K Svartberg, unpublished data) and may be a more common behavioral problem than has been acknowledged. Floor fearfulness appears to be more common in some breeds, and especially the collie seems to be over-represented in this respect (K Svartberg, unpublished data). In this paper, we will focus on the physiological changes that are associated with behaviorally expressed fear in two fear-eliciting tests, a floor test and a gunshot test.

\section{Materials and Methods}

\section{The dogs}

Fourteen privately owned male dogs of the collie breed (3-10 years old) were recruited for the study. The dogs were selected by means of a questionnaire, in which the dog owners gave their opinion on whether their dogs were fearful of gunshots and other noise, and whether they were fearful of walking on different types of floor. Seven dogs said to be fearful of gunshots and seven dogs said to be fearless of gunshots were selected. This sample of 14 individuals also included seven individuals that were said to be fearful of floors and seven dogs claimed to be fearless of floors. Blood sampling turned out to be impossible in one dog and this animal was therefore excluded from the study. During the study the dogs were not allowed to eat and drink. The study was carried out on 10 separate days and each dog went through the test alone without any contact with other dogs. The use of privately owned dogs was approved by the National Board of Agriculture, and the experimental procedure was approved by the Animal Ethics Committee in Uppsala, Sweden.

\section{Experimental procedure}

When the dogs arrived at the test place, a heart rate monitor was strapped on. Heart rate was measured using the Polar Sport tester for human beings (Polar Vantage NV; Polar Ltd, Bromma, Sweden). In order to maximize the contact between the electrodes and the skin, electrode gel (Blågel; Cefar Medical Products AB, Lund, Sweden) was applied to a small area behind the left foreleg. Then the belt was put around the chest and fastened with Vetrap (3 M Animal Care Products, St Paul, MN, USA) to further increase the contact. The heart rate was registered every $5 \mathrm{~s}$ and a mean value for every minute was calculated. After the heart rate monitor was fitted, a permanent catheter (SweVet; Piab, Sjöbo, Sweden) was inserted under local anesthesia (Xylocain; Astra, Södertälje, Sweden) in the cephalic vein. The catheter was fixed and kept in place with Vetrap. After insertion of the catheter, the dog was allowed to rest with its owner for $30 \mathrm{~min}$ before the floor test started.

The floor test was divided into three parts: 'floor 1', 'stairs', and 'floor 2'. 'Floor 1' included passages on plastic and parquet floors. The 'stairs' consisted of an open marble spiral staircase. 'Floor 2' included passages on clinker, concrete, iron grids, and wobbling boards. To standardize 
Table 1 Experimental procedure including blood sampling and heart rate monitoring

Heart rate recording

\section{Moment}

Heart rate equipment on;

insertion of catheter;

30 min rest

Blood sample 1 (30 min after insertion

of catheter)

Start plastic floor

Finish plastic floor

Start parquet floor

Finish parquet floor

Start stairs

Finish stairs

Blood sample 2

Start clinker floor

Finish clinker floor

Start concrete floor

Finish concrete floor

Start iron grids

Finish iron grids

Start wobbling boards

Finish wobbling boards

Blood sample 3

Walk to fenced meadow, 30 min rest

Blood sample 4

Shot 1

Shot 2 ( 5 min after shot 1$)$

Blood sample 5 (5 min after shot 2)

Blood sample 6 (15 min after shot 2)

Blood sample 7 (30 min after shot 2)

Blood sample 8 (60 min after shot 2)

Removal of catheter and heart rate

equipment off

the procedure, the same person, the test leader, held all dogs on a $2.3 \mathrm{~m}$ long leash during the test. The test leader, together with the dog owner, walked at an even and relatively slow pace. If the dog balked or tried to withdraw, the test leader and handler stopped walking for $10 \mathrm{~s}$. If the dog did not start to walk by itself, the dog was verbally encouraged to follow by the handler and test leader. If the dog refused to walk, the test leader gently forced the dog using the leash. If the dog still refused to walk after $30 \mathrm{~s}$, the test was interrupted. Blood samples 1 and 2 were taken before the start of the first and second floor tests respectively, and sample 3 was taken when the floor test was completed (Table 1). The dogs' behavior was video recorded by a hand-held camera for later analyses.

After the floor test, the (leashed) dog was walked by the dog owner to a large $(\mathrm{ca} .80 \times 80 \mathrm{~m})$ fenced meadow and rested there alone with the dog owner and test leader for $30 \mathrm{~min}$ before the fourth blood sample was taken. The gunshot test was then started. During this test, the dog stood beside its owner, leashed on a $40 \mathrm{~m}$ long cord (as a precaution in case the dog wanted to escape from the enclosure). A person hidden behind a tree $50 \mathrm{~m}$ from the dog fired two shots from a starter's gun, with $5 \mathrm{~min}$ between the gunshots. During and after the shots, the dog owner remained passive. If the dog tried to escape, it was free to do so within the enclosure without any action being taken. Five minutes after the second shot, the fifth blood sample was taken. The sixth, seventh, and eighth blood samples were taken 15, 30, and $60 \mathrm{~min}$ after the second shot (Table 1). The test was now completed and the catheter and heart rate equipment were removed. The whole procedure took about $4 \mathrm{~h}$ for each dog depending on how fast the dog carried out the floor tests.

Before the experiment started, the dog's reaction to a stranger (the test leader) and interest in playing with the stranger were tested according to a standardized behavioral test used by the Swedish Working Dog Association, the 'Dog Mentality Assessment' (Svartberg \& Forkman 2002). The interest in playing was repeated after the floor test (after the third blood sample) and after the gunshot test (after the fifth blood sample) (Table 1). However, results from these tests are not presented in the present paper.

\section{Classification of dogs according to fearfulness of floors and gunshots}

After the tests, the dogs' behavioral reactions were coded according to the video recordings. In the three parts of the floor test (floor 1, stairs, floor 2), the dogs were ranked according to hesitation to walk and to whether they walked close to the wall. These variables represented measures of immobility and avoidance/escape respectively, which are common fear-related behaviors in the dog. Hesitation to walk was scored from 1 to 4 , where $1=$ no hesitation at all, $2=$ some hesitation (i.e. the dog slows down when coming out on a new surface), 3 =hesitates before stepping out on the surface, and $4=$ refuses to walk. Walking close to the wall was also scored from 1 to 4 , where $1=$ no tendency to move towards the wall, $2=a$ tendency to move towards the wall, $3=$ a tendency to walk close to the wall, and $4=$ walking close to the wall. Dogs which scored a mean value of $\geq 2$ in total in all three parts of the floor test were categorized as 'floor fearful', while dogs which scored $<2$ were classified as 'floor fearless'. In the gunshot test, the dogs' initial startle reaction during both shots were scored from 1 to 5 , where $1=$ no reaction, or looks up, $2=$ increased activity, but no escape, $3=$ tends to escape, $4=$ escapes a short distance and stops, and $5=$ escapes a long distance with no stop. In addition, the dogs' degree of fear during both shots one and two was scored from 1 to 5 , where $1=$ no fear, $2=$ slight fear, $3=$ obvious fear, $4=$ very frightened, and $5=$ terrified. Dogs that scored a mean value of $\geq 2$ in all parts of the gunshot test were classified as 'gun fearful', while dogs that scored $<2$ were classified as 'gun fearless'. 
Blood plasma analyses

Blood was withdrawn into ice-chilled tubes containing EDTA tripotassium salt and aprotinin (Trasylol; Bayer, Leverkusen, Germany; 2500 kallikrein inhibitor units/ $5 \mathrm{ml}$ tube). The hematocrit level was determined by centrifuging the blood in capillary tubes for $5 \mathrm{~min}$ (ALC Centrifugette 4203; Skafte \& Claesson AB, Göteborg, Sweden). Thereafter, the blood was centrifuged at $1500 \mathrm{~g}$ for $20 \mathrm{~min}$ at $4{ }^{\circ} \mathrm{C}$, and stored at $-20^{\circ} \mathrm{C}$ for $24 \mathrm{~h}$ and then at $-80{ }^{\circ} \mathrm{C}$ until assayed. Dilutions of canine plasma were parallel to the standard curve in all RIAs described below. Plasma cortisol, progesterone, and testosterone were measured using the Coat-a-Count RIA (Diagnostic Product Corporation, Los Angeles, CA, USA). Plasma vasopressin and $\beta$-endorphin were extracted and analyzed with the Euro-Diagnostica AB RIA (Ideon, Malmö, Sweden). The lower detection limit was $2.9 \mathrm{nmol} / 1$ for cortisol, $0.1 \mathrm{nmol} / 1$ for progesterone, $0.05 \mathrm{nmol} / 1$ for testosterone, $0.7 \mathrm{pmol} / 1$ for vasopressin, and $3.6 \mathrm{pmol} / 1$ for $\beta$-endorphin. In all four RIAs, the samples were measured in the same assay. The intra-assay coefficient of variance for cortisol was $<10 \%$ between 23 and $1380 \mathrm{nmol} / 1$, for progesterone $<10 \%$ between 1 and $134 \mathrm{nmol} / 1$, for testosterone $<10 \%$ between 0.7 and $55 \mathrm{nmol} / 1$, for vasopressin $<10 \%$ between 1.4 and $60 \mathrm{pmol} / 1$, and for $\beta$-endorphin $<10 \%$ between 5 and $62 \mathrm{pmol} / \mathrm{l}$.

\section{Statistical analysis}

Values are presented as means \pm S.E.M. Data were analyzed using the SAS software (SAS Institute Inc. 1996). For comparisons between the groups, the blood variables were divided into three blocks, namely, sample 1 (control), samples 2-4 (floor test), and samples 5-8 (gunshot test), and the heart rate recordings into recording 1 (control), recordings 2-18 (floor test), and recordings 19-24 (gunshot test). The repeated measurement ANOVA (MIXED procedure) was applied to the different parameters. The statistical model included the fixed effect of sample and the random effect of dog. Pairwise comparisons with the first sample were tested for significance using differences in least square means (the DIFF option). The significance level was set at $P \leq 0 \cdot 05$.

\section{Results}

\section{Classification of dogs according to fearfulness of floors and} gunshots

According to our behavioral criteria, seven of the dogs were classified as fearful of floors $(\operatorname{dogs} 3,4,5,6,8,9,10$, ranking value $3 \cdot 1$ (range $2-3 \cdot 2)$ ) and six were not (dogs 1 , $2,7,11,12,13$, ranking value $1 \cdot 1$ (range $1 \cdot 0-1 \cdot 4)$ ), seven dogs were classified as fearful of gunshots $(\operatorname{dogs} 2,3,4,5$,
$6,7,9$, ranking value $2 \cdot 8$ (range $2 \cdot 0-4 \cdot 5)$ ) and six were not (dogs $1,8,10,11,12,13$, ranking value $1 \cdot 2$ (range $1 \cdot 0-1 \cdot 5)$ ). Five dogs were classified as fearful of both floors and gunshots and four were classified as fearless in both tests. The same dogs that were judged to be fearful of gunshots by their owners were also classified as fearful according to our test results. However, the classification of fearfulness of walking on certain floors, according to the owners' judgment, did not entirely correlate with our classification following the floor tests. In this paper, we only use the classification made according to our test. Since some of the dogs were afraid of floors, but not of gunshots, and vice versa, we present the results from the floor and gunshot test first according to the dogs' response to floors (Figs 1-7a) and thereafter according to their response to gunshots (Figs 1-7b).

\section{Heart rate and hematocrit}

Classification according to floor fearfulness The heart rate increased significantly during the floor test compared with the first value in both fearful and fearless dogs. Dogs fearful of floors had significantly higher heart rates during the floor test than did fearless dogs. There were no differences between the groups before the test started and during the gunshot test (Fig. 1a).

In dogs fearful of floors, the hematocrit value increased after the stairs in the floor test and after the shots, but only remained elevated $15 \mathrm{~min}$ after the shots in dogs fearless of floors. There were no significant differences in the hematocrit concentration between the groups (Fig. 2a).

Classification according to gunshot fearfulness In both groups of dogs, the heart rate increased significantly, compared with the first value, during the floor test and in single samples during the gunshot test. Dogs fearful of gunshots had significantly higher heart rates, both during the floor test and after the shots, compared with gunshotfearless dogs (Fig. 1b).

Compared with the first sample, the hematocrit increased significantly in dogs fearful of gunshots, both during the floor test and after the shots. In gunshot-fearless dogs, the hematocrit concentration was only increased after the stairs. Dogs fearful of gunshots had significantly higher hematocrit values after the gunshots compared with gunshot-fearless dogs, but there was no significant difference between the groups before the test started and during the floor test (Fig. 2b).

\section{Cortisol and progesterone concentrations}

Classification according to floor fearfulness Neither in floor-fearful nor in floor-fearless dogs did the plasma cortisol concentration change during the floor test, but it did increase significantly after the shots. However, there were no significant differences between the groups (Fig. 3a). 
(a)

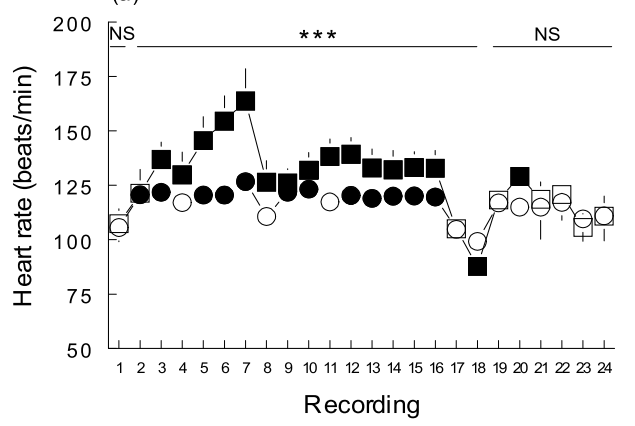

(b)

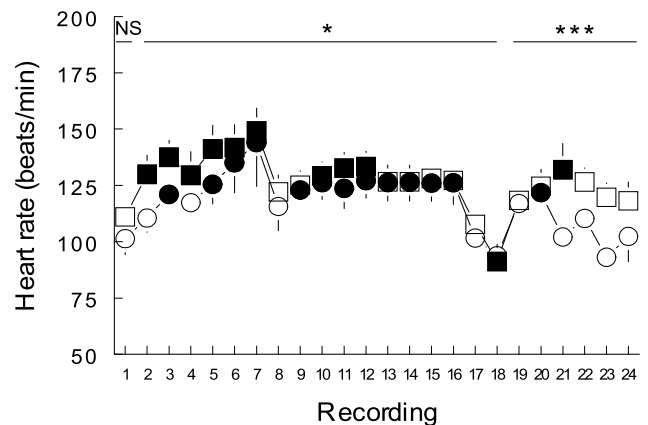

Figure 1 Heart rate during a floor test and a gunshot test in 13 dogs. Seven of the dogs were fearful of floors (squares) and six were fearless of floors (circles) (a), and seven dogs were fearful of gunshots (squares) and six were fearless of gunshots (circles) (b). For details of the recordings see Table 1 . Values are presented as means \pm S.E.M. Solid symbols indicate that values are significantly different from recording $1(P \leq 0 \cdot 05)$. For comparisons between the groups, the recordings were divided into three blocks, recording 1 , recordings $2-18$ (floor test), and recordings 19-24 (gunshot test). Dogs that were fearful of floors had a significantly higher heart rate during the floor test than dogs fearless of floors. There were no significant differences before the test or during the gunshot test (a). Dogs that were fearful of gunshots had significantly elevated heart rates during the floor test and gunshot test compared with dogs fearless of gunshots. There were no significant differences before the test started (b). NS, not significant, ${ }^{*} P \leq 0 \cdot 05,{ }^{* * *} P \leq 0 \cdot 001$.

There was no change in progesterone concentration during the floor test, but a significant increase in progesterone was seen after the shots, both in fearful and in fearless dogs. There were no significant differences between the groups (Fig. 4a).

Classification according to gunshot fearfulness In dogs fearful of shots, the plasma cortisol concentration did not change during the floor test, but a significant increase was seen immediately after the shots and the concentration was still elevated in the last blood sample. There were no significant changes in gunshot-fearless dogs. Dogs fearful of (a)

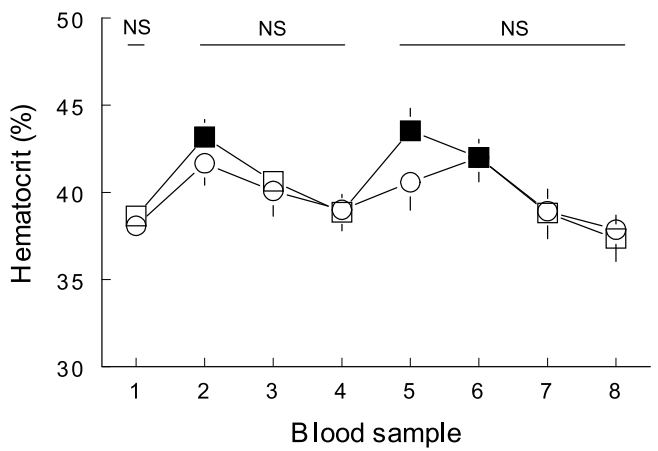

(b)

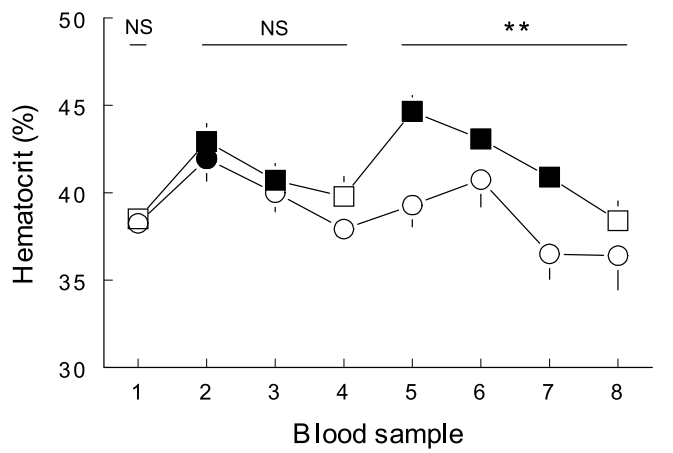

Figure 2 Hematocrit during a floor test and a gunshot test in 13 dogs. Seven of the dogs were fearful of floors (squares) and six were fearless of floors (circles) (a), and seven dogs were fearful of gunshots (squares), while six were fearless of gunshots (circles) (b). For details of the sampling see Table 1. Values are given as means \pm S.E.M. Solid symbols indicate that values are significantly different from sample $1(P \leq 0 \cdot 05)$. For comparisons between the groups, the samples were divided into three blocks, sample 1, samples 2-4 (floor test), and samples 5-8 (gunshot test). There were no significant differences in hematocrit concentration between dogs fearful and dogs fearless of floors (a). Dogs that were fearful of gunshots had significantly elevated hematocrit values during the gunshot test compared with dogs fearless of gunshots. There were no significant differences between the groups before the test started and during the floor test (b). NS, not significant, ${ }^{* *} P \leq 0 \cdot 01$.

gunshots had significantly higher cortisol concentrations after the shots compared with fearless dogs. There were no significant differences between the groups before the tests started and during the floor test (Fig. 3b).

Plasma progesterone did not change during the floor test, but increased significantly after the shots in gunshotfearful dogs. The progesterone concentration after the shots was significantly higher in gunshot-fearful dogs than it was in gunshot-fearless dogs. There were no significant differences in progesterone between the groups before the tests started and during the floor test (Fig. 4b). 
(a)

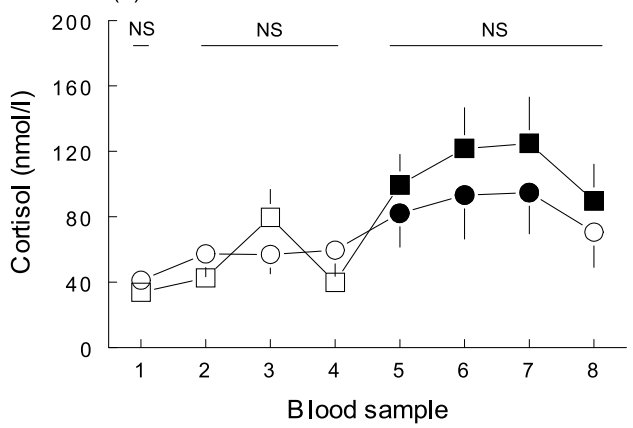

(b)

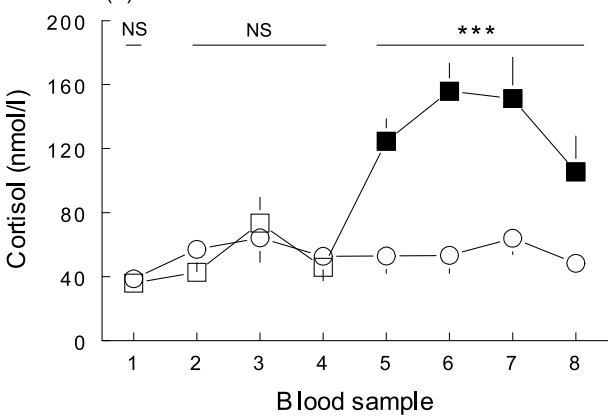

Figure 3 Plasma cortisol during a floor test and a gunshot test in 13 dogs. Seven of the dogs were fearful of floors (squares) and six were fearless of floors (circles) (a), while seven dogs were fearful of gunshots (squares) and six were fearless of gunshots (circles) (b). For details of the sampling see Table 1. Values are presented as means \pm S.E.M. Solid symbols indicate that values are significantly different from sample $1(P \leq 0 \cdot 05)$. For comparisons between the groups, the samples were divided into three blocks, sample 1 , samples 2-4 (floor test), and samples 5-8 (gunshot test). There were no significant differences during the experiment between dogs fearful and dogs fearless of floors (a). Dogs that were fearful of gunshots had significantly higher cortisol concentrations during the gunshot test than dogs that were fearless of gunshots. There were no significant differences between the groups before the test started and during the floor test (b). NS, not significant, $* * * P \leq 0 \cdot 001$.

Testosterone, vasopressin and $\beta$-endorphin concentrations

Classification according to floor fearfulness The testosterone concentration increased in fearful and fearless dogs in the fourth and the fifth blood samples respectively. There were no significant differences between the groups (Fig. 5a).

In both fearful and fearless dogs, the vasopressin concentration increased significantly, compared with the first value, in the fourth and the sixth blood samples respectively. Dogs fearful of floors had significantly higher vasopressin concentrations during the gunshot test compared with fearless dogs. There were no significant differences regarding vasopressin between the groups before the tests started and during the floor test (Fig. 6a). (a)

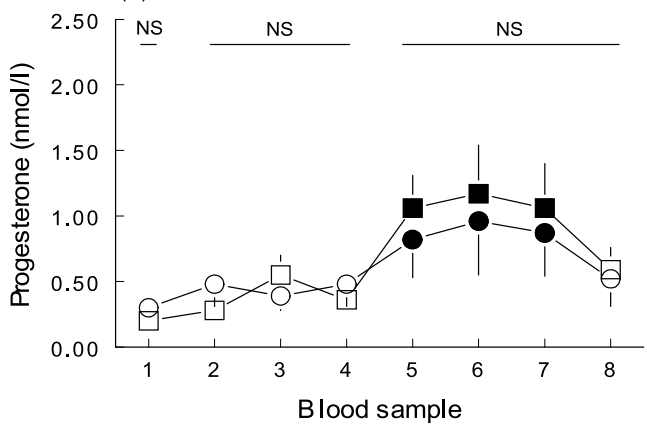

(b)

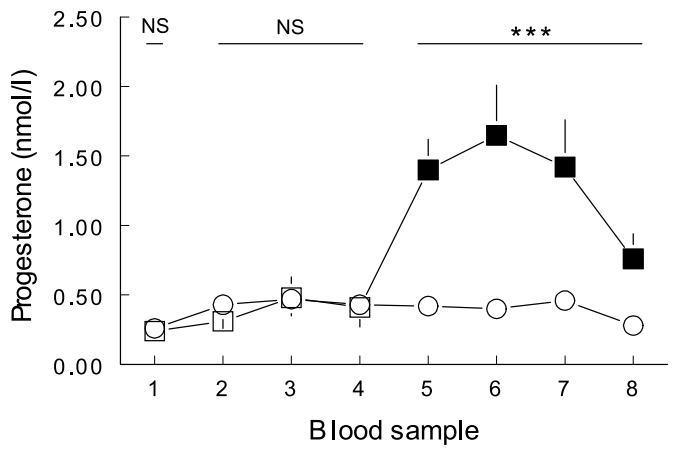

Figure 4 Plasma progesterone during a floor test and a gunshot test in 13 dogs. Seven of the dogs were fearful of floors (squares) and six were fearless of floors (circles) (a), and seven dogs were fearful of gunshots (squares) and six were fearless of gunshots (circles) (b). For details of the sampling see Table 1. Values are presented as means \pm S.E.M. Solid symbols indicate that values are significantly different from sample $1(P \leq 0 \cdot 05)$. For comparisons between the groups, the samples were divided into three blocks, sample 1, samples 2-4 (floor test), and samples 5-8 (gunshot test). There were no significant differences during the experiment between dogs fearful and dogs fearless of floors (a). Dogs that were fearful of gunshots had a significantly higher progesterone concentration during the gunshot test than dogs did that were fearless of gunshots. There were no significant differences between the groups before the test started and during the floor test (b). NS, not significant, ${ }^{* *} P \leq 0 \cdot 001$.

Compared with the first sample, the $\beta$-endorphin concentration increased significantly during the floor test and after the shots in dogs fearful of floors. In fearless dogs, the concentration was significantly increased only in the last sample. However, there were no significant differences between the groups (Fig. 7a).

Classification according to gunshot fearfulness The plasma testosterone concentration increased in fearful and fearless dogs in the fourth and fifth blood samples respectively, and stayed high until the last blood sample. There were no significant differences between the groups (Fig. 5b). 
(a)

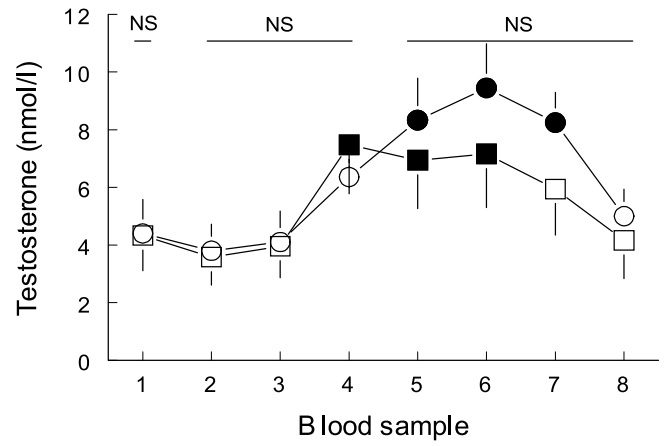

(b)

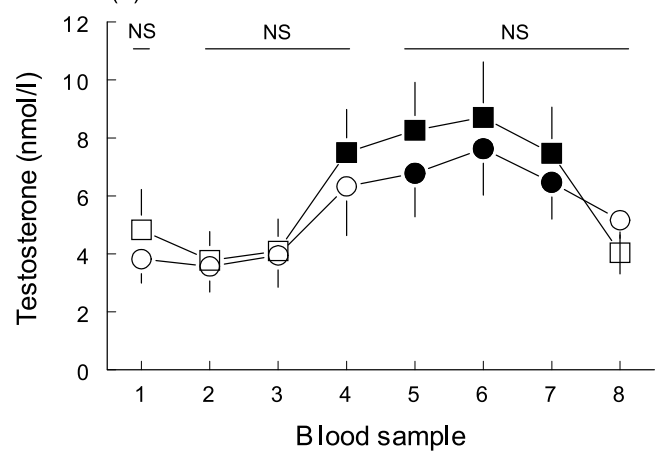

Figure 5 Plasma testosterone during a floor test and a gunshot test in 13 dogs. Seven of the dogs were fearful of floors (squares) and six were fearless of floors (circles) (a), while seven dogs were fearful of gunshots (squares) and six were fearless of gunshots (circles) (b). For details of the sampling see Table 1. Values are given as mean values \pm S.E.M. Solid symbols indicate that values are significantly different from sample $1(P \leq 0 \cdot 05)$. For comparisons between the groups, the samples were divided into three blocks, sample 1, samples 2-4 (floor test), and samples 5-8 (gunshot test). There were no significant differences during the experiment between dogs fearful and dogs fearless of floors (a). Neither were there any significant differences between the groups of dogs fearful and dogs fearless of gunshots (b). NS, not significant.

In both fearful and fearless dogs, the vasopressin concentration increased significantly, compared with the first value, in the fourth and the fifth blood samples respectively. Dogs that were fearful of gunshots had significantly higher vasopressin levels during the gunshot test compared with fearless dogs. There were no significant differences between the groups before the tests started and during the floor test (Fig. 6b).

The $\beta$-endorphin concentration increased significantly in comparison with the first sample during the floor test and after the shots in dogs fearful of gunshots. In fearless dogs, no significant changes were seen. After the gunshots, dogs that were fearful of gunshots had significantly higher $\beta$-endorphin levels than fearless dogs. There were no (a)

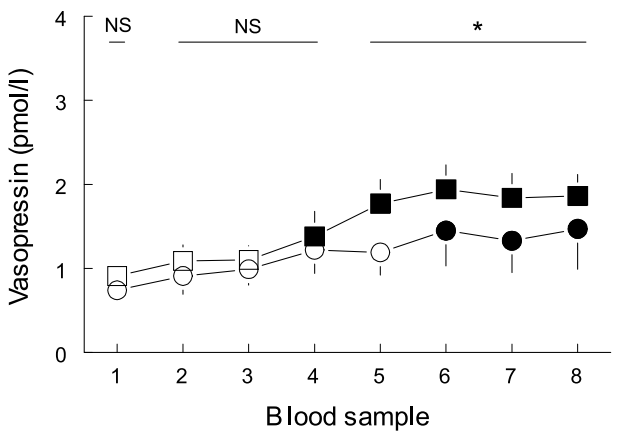

(b)

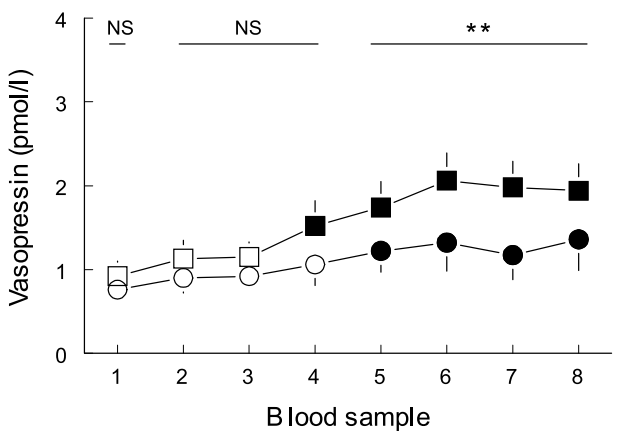

Figure 6 Plasma vasopressin during a floor test and a gunshot test in 13 dogs. Seven of the dogs were fearful of floors (squares) and six were fearless of floors (circles) (a), while seven dogs were fearful of gunshots (squares) and six were fearless of gunshots (circles) (b). For details of the sampling see Table 1. Values are given as mean values \pm S.E.M. Solid symbols indicate that values are significantly different from sample $1(P \leq 0 \cdot 05)$. For comparisons between the groups, the samples were divided into three blocks, sample 1, samples 2-4 (floor test), and samples 5-8 (gunshot test). Dogs that were fearful of floors had a significantly higher vasopressin concentration during the gunshot test than dogs fearless of floors. There were no significant differences between the groups before the test started and during the floor test (a). Dogs that were fearful of gunshots had a significantly higher vasopressin concentration during the gunshot test than dogs did that were fearless of gunshots. There were no significant differences between the groups before the test started and during the floor test (b). NS, not significant, ${ }^{\star} P \leq 0 \cdot 05,{ }^{*} P \leq 0 \cdot 01$.

significant differences between the groups before the tests started and during the floor test (Fig. 7b).

\section{Discussion}

To our knowledge, this is the first time that dogs that are behaviorally fearful of gunshots have been reported to have increased heart rate, hematocrit level, and plasma concentrations of cortisol, progesterone, vasopressin, and endorphins, reaching significantly higher values during and after gunshots compared with fearless dogs. The results 
(a)

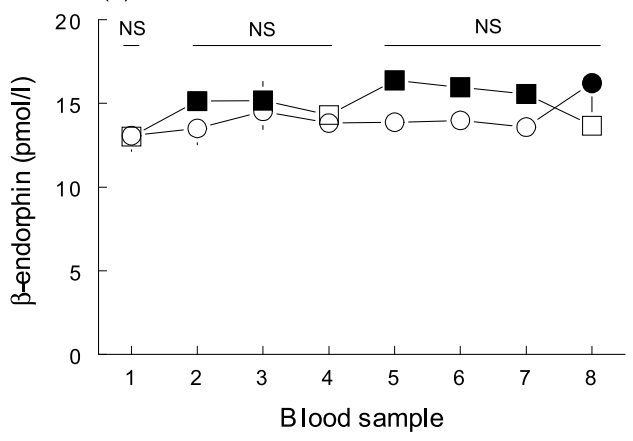

(b)

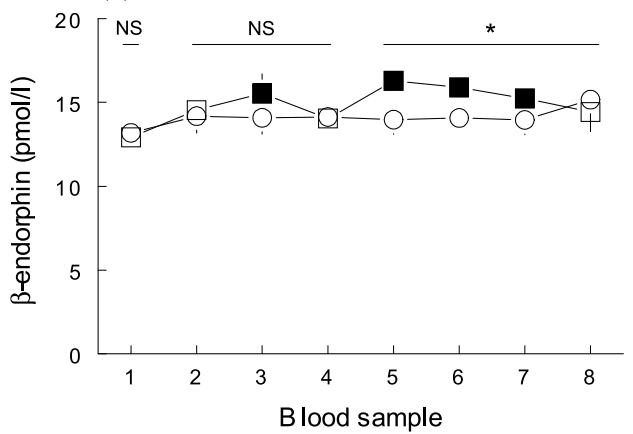

Figure 7 Plasma $\beta$-endorphin during a floor test and a gunshot test in 13 dogs. Seven of the dogs were fearful of floors (squares) and six were fearless of floors (circles) (a), and seven dogs were fearful of gunshots (squares) and six were fearless of gunshots (circles) (b). For details of the sampling see Table 1. Values are presented as means \pm S.E.M. Solid symbols indicate that values are significantly different from sample $1(P \leq 0 \cdot 05)$. For comparisons between the groups, the samples were divided into three blocks, sample 1, samples 2-4 (floor test), and samples 5-8 (gunshot test). There were no significant differences during the experiment between dogs fearful and dogs fearless of floors (a). Dogs that were fearful of gunshots had significantly elevated endorphin concentrations during the gunshot test compared with fearless dogs. There were no significant differences between the groups before the test started and during the floor test (b). NS, not significant, ${ }^{*} P \leq 0 \cdot 05$.

demonstrated that this type of fear affects all physiological stress systems, confirming that it constitutes serious stress for the individual. Most striking in the present study was the large increase in plasma cortisol and progesterone during the gunshot test in dogs that were fearful of gunshots, as well as the finding that the plasma concentrations of these hormones did not change in fearless dogs. The results indicate that progesterone may be a good alternative to cortisol for assessing stress in male dogs.

All dogs that were classified as fearful of gunshots, according to the results of our behavior test, were described as fearful of gunshots by their owners in the questionnaire. This demonstrates that most dog owners recognize problems with noises and gunshots. By contrast, only some of the dogs that were classified as fearful of floors by us were known to be fearful of floors by their owners. There could be several explanations for this. For instance, there are numerous types of floors and the floor test result may depend on which types of floor the individual dog is used to. Most dogs accept walking on floors that exist in their home setting. For this reason, a floor test is more difficult to standardize than a gunshot test. Physiological differences between dogs that were fearful of walking on different floors and dogs that were fearless of floors were few. Among the variables tested, only the heart rate was higher during the floor test in dogs fearful of walking on floors. It is not surprising that heart rate increased during the floor test, since this variable has a tendency to increase in several stress situations, e.g. when dogs are exposed to sound blasts, shock, falling bags, opening umbrellas, and restraint (Galosy et al. 1979, Beerda et al. 1998). However, what was quite surprising is that the heart rate remained elevated during the entire floor test. Since stress and emotional stimuli are known to cause prompt increases in heart rate followed, however, by rapid decreases (CaraffaBraga et al. 1973), our study demonstrated that the sympathetic nervous system became activated for a long period, which was partly confirmed by the increased hematocrit level. In earlier stress studies in dogs, the hematocrit level did not increase significantly during transportation (Kuhn et al. 1991). Furthermore, DeLuca et al. (1974) did not find any differences in hematocrit levels between normal and nervous strains of dogs. Thus, fearfulness of floors seems to trigger a predominant sympathetic response, whereas fearfulness of gunshots may be a more classical hypothalamic-pituitary-mediated response.

Interestingly, in contrast to the other blood variables, fearful dogs had increased testosterone and vasopressin concentrations already by the fourth blood sample, before the gunshot tests started. After the third sample, the dogs went to the fenced meadow, leashed, and were allowed to rest for $30 \mathrm{~min}$ before the fourth blood sample was taken and the gunshot test started. Increases in testosterone and vasopressin have been reported to be related to physical activity (Campbell et al. 1982, Wade 1984), which may explain the fact that the increases had already started before the gunshot test took place. In addition, it has been reported that stimulation with ACTH does not increase testosterone concentrations in male dogs, but does in females (Frank et al. 2003), which may be an additional explanation as to why testosterone levels did not change further in response to the tests in this study.

There were no differences between the control samples in any of the measured variables between fearful and fearless dogs. This suggests that the physiological variables in all groups of dogs were at the same level before the experiment started. It could be argued that the dogs' reactions to the floor test may have influenced the results 
of the later gunshot test. However, since all levels tested, except testosterone and vasopressin, returned to baseline before the gunshot test started, this indicates that the increased physiological variables during the gunshot test were due to the gunshots. When using physiologically invasive methods, it is always important to evaluate the effect of the sampling per se. It is important not to stress the dogs with the sampling. Earlier studies have shown that petting during the blood sampling may inhibit the plasma cortisol response following venipuncture in dogs (Hennessy et al. 1998). Other studies in dogs have shown that variables such as testosterone are not influenced by blood sampling (Knol et al. 1992). It is difficult to speculate on the higher vasopressin and $\beta$-endorphin concentrations during the gunshot test in dogs fearful of gunshots. The higher values may have been caused by pain in their ears, but may also simply have been an effect of the whole stress situation. Further studies are required to investigate why some dogs express such fear of gunshots and noises and some do not.

We conclude that dogs fearful of gunshots have increased levels of several physiological variables during gunshots, showing that this fear presents a serious stress to the individual. Since fears and mentality problems are common in dogs, studying physiological changes may be an additional tool in selecting breeding animals and working dogs. Furthermore, the results show that behaviorally expressed fear in the $\operatorname{dog}$ is accompanied by physiological changes associated with stress. This suggests that behavioral measurements are reliable in assessments of fearfulness and stress in the dog.

\section{Acknowledgements}

This work was supported by the Scientific Council for Medicine (grant no. 3392). We gratefully acknowledge all participating dogs and dog owners, Birgitta Åhman and the late Kicki Anerland for help with recruiting the dogs, Åsa Eriksson and Inger Lind for helping us with the practicalities, Gunilla Drugge-Boholm and Gunilla Ericson-Forslund for excellent help with hormonal analyses, and Professor Kerstin Olsson for valuable criticism of the manuscript.

\section{References}

Beaver BV 1999 Canine Behaviour: A Guide for Veterinarians. Philadelphia, PA, USA: WB Saunders Company.

Beerda B, Schilder MBH, Van Hooff JARAM, De Vries HW \& Mol JA 1998 Behavioural, saliva cortisol and heart rate responses to different types of stimuli in dogs. Applied Animal Behaviour Science 58 365-381.

Beerda B, Schilder MBH, Bernadina W, Van Hooff JARAM, De Vries HW \& Mol JA 1999 Chronic stress in dogs subjected to social and spatial restriction. II. Hormonal and immunological responses. Physiology and Behavior 66 243-254.
Beerda B, Schilder MBH, Van Hooff JARAM, De Vries HW \& Mol JA 2000 Behavioural and hormonal indicators of enduring environmental stress in dogs. Animal Welfare 9 49-62.

Bergeron R, Scott SL, Emond J-P, Mercier F, Cook NJ \& Schaefer AL 2002 Physiology and behavior of dogs during air transport. Canadian Journal of Veterinary Research 66 211-216.

Bernhardt PC, Dabbs JM, Fielden JA \& Lutter CD 1998 Testosterone changes during vicarious experiences of winning and losing among fans at sporting events. Physiology and Behavior 65 59-62.

Blackshaw JK 1988 Abnormal behaviour in dogs. Australian Veterinary Journal 65 393-394.

Boissy A 1995 Fear and fearfulness in animals. Quarterly Review of Biology 70 165-191.

Cameron V, Espiner E, Nicholls M, Donald R \& MacFarlane M 1985 Stress hormones in blood and cerebrospinal fluid of conscious sheep: effect of hemorrhage. Endocrinology 116 1460-1465

Campbell IT, Walker RF, Riad-Fahmy D, Wilson DW \& Griffiths K 1982 Circadian rhythms of testosterone and cortisol in saliva: effects of activity-phase and continuous daylight. Chronobiologia 9 389-396.

Caraffa-Braga E, Granata L \& Pinotti O 1973 Changes in blood-flow distribution during acute emotional stress in dogs. Pflugers Archiv. European Journal of Physiology 339 203-216.

Christiansen K \& Knussmann R 1987 Androgen levels and components of aggressive behavior in men. Hormones and Behavior 21 170-180.

Christiansen K, Knussmann R \& Couwenbergs C 1985 Sex hormones and stress in the human male. Hormones and Behavior 19 426-440.

Dabbs JM 1993 Salivary testosterone measurements in behavioral studies. Annals of the New York Academy of Sciences 20 177-183.

Davies RH, Harris B, Thomas DR, Cook N, Read G \& Riad-Fahmy D 1992 Salivary testosterone levels and major depressive illness in men. British Journal of Psychiatry 161 629-632.

DeLuca DC, Murphree OD \& Angel C 1974 Biochemistry of nervous dogs. Pavlovian Journal of Biological Science 9 136-148.

Dess NK, Linwick D, Patterson J \& Overmier JB 1983 Immediate and proactive effects of controllability and predictability on plasma cortisol responses to shocks in dogs. Behavioral Neuroscience $\mathbf{9 7}$ 1005-1016.

Fox MW 1978 The Dog: its Domestication and Behavior. New York: Garland STPM Press.

Frank LA, Rohrbach BW, Bailey EM, West JR \& Oliver JW 2003 Steroid hormone concentration profiles in healthy intact and neutered dogs before and after cosyntropin administration. Domestic Animal Endocrinology 24 43-57.

Fredrickson R \& Geary L 1982 Endogenous opioid peptides: review of physiological, pharmacological and clinical aspects. Progress in Neurobiology 19 19-69.

Galosy RA, Clarke LK \& Mitchell JH 1979 Cardiac changes during behavioral stress in dogs. American Journal of Physiology 236 H750-H758.

Giusto ELD, Cairncross K \& King MG 1971 Hormonal influences on fear-motivated responses. Psychological Bulletin 75 432-444.

Gray JA 1987 The Psychology of Fear and Stress, edn 2. Cambridge: Cambridge University Press.

Hennessy MB, Williams MT, Miller DD, Douglas CW \& Voith VL 1998 Influence of male and female petters on plasma cortisol and behaviour: can human interaction reduce the stress of dogs in a public animal shelter? Applied Animal Behaviour Science 61 63-77.

Henry J 1993 Biological basis of the stress response. News in Physiological Sciences 8 69-73.

King T, Hemsworth PH \& Coleman GJ 2003 Fear of novel and startling stimuli in domestic dogs. Applied Animal Behaviour Science 82 45-64.

Knol BW, Dieleman SJ, Bevers MM, Van den Brom WE \& Molt JA 1992 Effects of methods used for blood collection on plasma concentrations of luteinising hormone, testosterone, and cortisol in male dogs. Veterinary Quarterly 14 126-129. 
Kuhn G, Lichtwald K, Hardegg W \& Abel HH 1991 The effect of transportation stress on circulating corticosteroids, enzyme activities and hematological values in laboratory dogs. Journal of Experimental Animal Science 34 99-104.

Lindsay SR 2001 Handbook of Applied Dog Behaviour and Training, vol 2. Etiology and Assessment of Behavioral Problems. Ames: Iowa State University Press.

McCaul KD, Gladue BA \& Joppa M 1992 Winning, losing, mood, and testosterone. Hormones and Behavior 26 486-504.

Molina PE \& Abumrad NN 1994 Metabolic effects of opiates and opioid peptides. Advances in Neuroimmunology 4 105-116.

Müller MJ 1994 Salivary testosterone and simply reaction time parameters. Neuropsychobiology 30 173-177.

Plutchik R 1971 Individual and breed differences in approach and withdrawal in dogs. Behaviour 40 302-311.

Reeve EB, Gregerson MI, Allen TH \& Sear H 1953 Distribution of cells and plasma in the normal and splenectomised dog and its influence on blood volume with P32 and T-1824. American Journal of Physiology 175 195-203.

SAS Institute Inc. 1996 SAS User's Guide. Cary: SAS.

Scerbo AS \& Kolko DJ 1994 Salivary testosterone and cortisol in disruptive children: relationship to aggressive, hyperactive, and internalizing behaviors. Journal of the American Academy of Child and Adolescent Psychiatry 33 1174-1184.

Scott JP \& Fuller JL 1965 Genetics and the Social Behavior of the Dog. Chicago: University of Chicago Press.

Svartberg K \& Forkman B 2002 Personality traits in the domestic dog (Canis familiaris). Applied Animal Behaviour Science 79 133-155.

Van Honk J, Tuiten A, Verbaten R, Van den Hout M, Koppeschaar H, Thijssen J \& De Haan E 1999 Correlations among salivary testosterone, mood, and selective attention to threat in humans. Hormones and Behavior 36 17-24.

Wade C 1984 Response, regulations, and actions of vasopressin during exercise: a review. Medicine and Science in Sports and Exercise 16 $506-511$.

Received 18 June 2003

Accepted 11 December 2003

Made available online as an

Accepted Preprint 15 December 2003 